\title{
Planktonic stages of Processa macrodactyla (Decapoda: Caridea: Processidae) reared in the laboratory
}

\author{
A. Ortega*, H. Queiroga ${ }^{\dagger}$ and J.I. González-Gordillo** \\ * Centro Andaluz de Ciencia y Tecnología Marinas, Universidad de Cádiz, Campus Río San Pedro, E-11510 Puerto Real (Cádiz), Spain. \\ ${ }^{\dagger}$ Departamento de Biologia, Universidade de Aveiro, Campus de Santiago, 3800-193 Aveiro, Portugal. \\ ${ }^{\ddagger}$ Corresponding author, e-mail: JIGG, nacho.gonzalez@uca.es
}

\begin{abstract}
Two ovigerous females of the processid shrimp Processa macrodactyla were caught in April 1997 at $13 \mathrm{~m}$ depth in coastal waters of Rota, Cádiz Bay, south-western Spain $\left(36^{\circ} 36^{\prime} \mathrm{N} 6^{\circ} 18^{\prime} \mathrm{W}\right)$. Rearing was terminated after nine zoeal stages, when larvae moulted to the first juvenile instar. Descriptions of the appendages of every instar have been made so as to: (1) compare the larval morphology with that of other previous described known species in the genus Processa (P. canaliculata, P. edulis, P. elegantula, P. modica, $P$. nouveli); and (2) with those larvae not ascribed to a certain species in order to facilitate the specific identification of unknown collected planktonic larvae. When describing $P$. macrodactyla some characters remained, with few exceptions, invariable in their setation form ZI to ZIV or ZV, to then change and maintain until the last zoeal stage. This can be due to intermediate moults, with the result that some larvae unite the characters of Stages ZIV and ZV and others those of ZV and ZVI.
\end{abstract}

\section{INTRODUCTION}

The caridean genus Processa comprises numerous small shrimps spread worldwide, of which ten species are recorded within European waters (d'Udekem d'Acoz, 1999): Processa edulis Risso, 1816, Processa modica Williamson, 1979, Processa nouveli Al-Adhub \& Williamson, 1975, Processa canaliculata Leach, 1815, Processa elegantula Nouvel \& Holthuis, 1957, Processa macrophthalma Nouvel \& Holthuis, 1957, Processa acutirostris Nouvel \& Holthuis, 1957, Processa robusta Nouvel \& Holthuis, 1957, Processa macrodactyla Holthuis, 1952 and Processa intermedia Holthuis, 1951. Processa edulis was subdivided by Nouvel \& Holthuis (Nouvel \& Holthuis, 1957) into three subspecies: P. edulis edulis, distributed in the Mediterranean, and $P$. edulis crassipes and $P$. edulis arcassonensis recorded in the Atlantic. Williamson \& Rochanaburanon, 1979, proposed P. modica divided into $P$. modica carolii from the Mediterranean and south-west Atlantic coasts of Spain and the Atlantic subspecies $P$. modica modica. The species $P$. nouveli is considered with the subspecies $P$. nouveli nouveli in the Mediterranean and $P$. nouveli holthuisi in the Atlantic (Al-Adhub \& Williamson, 1975). Processa acutirostris is only found in the Mediterranean and $P$. intermedia occurs in Atlantic waters, while $P$. canaliculata, $P$. elegantula, $P$. robusta and $P$. macrophthalma are recorded from Atlantic waters as well as in the western Mediterranean Sea (d'Udekem d'Acoz, 1999). Finally, P. macrodactyla appears on the south Atlantic coasts of Spain (González-Gordillo \& Rodríguez, 2000) and the Alboran Sea (García Raso \& Casanova, 1985).

The larval morphology of these species is scarcely known since only partial descriptions of zoeal stages are available for $P$. edulis (Gurney, 1923 as P. canaliculata: Z1-Z4 from plankton specimens; Lebour, 1936: Z8 from plankton samples; Kurian, 1956: Z1-Z7 from plankton samples; Fincham \& Williamson, 1978 as P. edulis crassipes: Z6 from plankton samples; Barnich,
1996 as $P$. edulis edulis, from plankton samples); $P$. modica (Fincham \& Williamson, 1978 as P. modica modica: Z5; Williamson \& Rochanaburanon, 1979 as $P$. modica modica: Z1-Z7 from plankton samples; Barnich, 1996 as $P$. modica carolii: Z8 from plankton samples), $P$. nouveli (Gurney, 1923 as P. canaliculata: Z5-Z9 from plankton specimens; Kurian, 1956 as P. canaliculata: Z1, Z5, Z6 from plankton specimens; Fincham \& Williamson, 1978 as $P$. nouveli holthuisi: Z1, Z6; Williamson \& Rochanaburanon, 1979 as $P$. nouveli holthuisi: Z1-Z9 from laboratory cultures; Barnich, 1996 as $P$. nouveli nouveli, from plankton samples), $P$. canaliculata (Lebour, 1936: Z1-Z5, Z8, Z9 from laboratory and plankton specimens; Fincham \& Williamson, 1978: Z6) and for P. macrodactyla (González-Gordillo \& Rodríguez, 2000: $\mathrm{Zl}$, hatched in the laboratory). For the other species of the genus no accurate larval descriptions are available. Other larval descriptions of processid species have been made from specimens collected in plankton samples from European waters although, at the present time, they remain unclassified as Processa ?elegantula, Processa sp.1, and Processa sp.2 (Barnich, 1996); Processa EM4 and Processa EM7 (Williamson, 1967); Processa EM5 and Processa EM6 (Williamson, 1967; dos Santos, 1999); and Processa EFSL11 (dos Santos, 1999).

In this paper, the complete larval development of P. macrodactyla was reared in the laboratory in order to describe and compare it with that of congeneric species with known larvae so as to facilitate the specific identification of unknown planktonic larvae.

\section{MATERIALS AND METHODS}

Two ovigerous shrimp were caught in April 1997 with a benthic trawl at $13 \mathrm{~m}$ depth in coastal waters of Rota, Cádiz Bay, south-western Spain $\left(36^{\circ} 36^{\prime} \mathrm{N} 6^{\circ} 18^{\prime} \mathrm{W}\right)$. The specimens were maintained in a 2-1 glass beaker, 
Table 1. Setation and other characteristics of zoeal stages of Processa macrodactyla. Setal groups on successive segments are separated by a comma and groups of setae on the same segment, or on different lobes of the same endite, are separated by a plus sign $(+)$. Carapace length in millimetres.

\begin{tabular}{|c|c|c|c|c|c|}
\hline \multirow[b]{2}{*}{ Features } & \multicolumn{5}{|c|}{ Stages } \\
\hline & Zoea I & Zoea II & Zoea III & Zoea IV & Zoea V \\
\hline Carapace length & $0.56 \pm 0.011$ & $0.61 \pm 0.017$ & $0.70 \pm 0.036$ & $0.80 \pm 0.055$ & $0.84 \pm 0.027$ \\
\hline \multicolumn{6}{|l|}{ Antennule } \\
\hline peduncle & naked & $2 p$ & $1 c+4 p, 1 e+7 p$ & $1 c+8 p, 1 e+8 p$ & $1 c+11 p, 3 e+4 p$ \\
\hline endopod & as long seta & as long seta & as long seta & as long seta & as long seta \\
\hline exopod & $1 p+1 s+3 a$ & $1 \mathrm{~s}+3 \mathrm{a}$ & $1 \mathrm{~s}+3 \mathrm{a}$ & $1 \mathrm{~s}+3 \mathrm{a}$ & $1 s+3 a$ \\
\hline \multicolumn{6}{|l|}{ Antenna } \\
\hline peduncle & $1 \mathrm{c}$ & $1 \mathrm{c}$ & $1 \mathrm{c}$ & $1 \mathrm{c}$ & $1 \mathrm{c}$ \\
\hline endopod & rows of spinules & rows of spinules & rows of spinules & rows of spinules & no spinules \\
\hline exopod & $9 p+1 j+2 p$ & $9 p+1 j+2 p$ & $12 p+1 j+1 p$ & $13 p+1 j+1 p$ & $14 p+1 j+1 p$ \\
\hline \multicolumn{6}{|l|}{ Maxillule } \\
\hline coxal endite & $2 e+5 d$ & $2 e+5 d$ & $2 e+5 d$ & $2 e+6 d$ & $8 \mathrm{e}$ \\
\hline basial endite & $5 \mathrm{t}$ & $7 \mathrm{t}$ & $8 \mathrm{t}$ & $8 \mathrm{t}$ & $10-11 \mathrm{t}$ \\
\hline endopod & $2 \mathrm{e}, 3 \mathrm{e}$ & $3 \mathrm{e}, 3 \mathrm{e}$ & $3 \mathrm{e}, 3 \mathrm{e}$ & $3 \mathrm{e}, 3 \mathrm{e}$ & $3 \mathrm{e}, 3 \mathrm{e}$ \\
\hline \multicolumn{6}{|l|}{ Maxilla } \\
\hline coxal endite & $(10 p)+(1 p+3 e)$ & $(11 p)+(2 p+2 e)$ & $(11 p)+(2 p+2 e)$ & $(11 p)+(2 p+2 e)$ & $(12 p)+(2 p+2 e)$ \\
\hline basial endite & $(1 p+3 e)+(1 p+3 e)$ & $(1 p+4 e)+(1 p+4 e)$ & $(1 p+4 e)+(1 p+4 e)$ & $(1 p+4 e)+(1 p+4 e)$ & $(1 p+5 e)+(1 p+5 e)$ \\
\hline endopod & $3 e+2 e+1 e+3 e$ & $3 e+2 e+1 e+3 e$ & $3 e+2 e+1 e+3 e$ & $3 e+2 e+1 e+3 e$ & $3 \mathrm{e}+2 \mathrm{e}, 1 \mathrm{e}+1 \mathrm{e}+2 \mathrm{e}$ \\
\hline scaphognathite & $3 p+2 p$ & $5 p+2 p$ & $7 p+2 p$ & $11 p+2 p$ & $17 p$ \\
\hline \multicolumn{6}{|l|}{ First maxilliped } \\
\hline coxa & $6 \mathrm{p}$ & $9 p$ & $9 p$ & $9 p$ & $9 p$ \\
\hline basis & $2 p+10 e$ & $2 p+12 e$ & $1 p+13 e$ & $1 p+13 e$ & $1 p+15 e$ \\
\hline endopod & $3 \mathrm{e}, 1 \mathrm{e}, 2 \mathrm{e}, 1 \mathrm{e}+3 \mathrm{e}$ & $3 \mathrm{e}, 1 \mathrm{e}, 2 \mathrm{e}, 1 \mathrm{e}+3 \mathrm{e}$ & $3 \mathrm{e}, 1 \mathrm{e}, 2 \mathrm{e}, 1 \mathrm{e}+3 \mathrm{e}$ & $3 \mathrm{e}, 1 \mathrm{e}, 2 \mathrm{e}, 1 \mathrm{e}+3 \mathrm{e}$ & $3 \mathrm{e}, 1 \mathrm{e}, 2 \mathrm{e}, 1 \mathrm{e}+3 \mathrm{e}$ \\
\hline exopod & $1 n+3 n$ & $\ln +4 n$ & $\ln +4 n$ & $\ln +4 n$ & $2 n+4 n$ \\
\hline \multicolumn{6}{|l|}{ Second maxilliped } \\
\hline coxa & $2 \mathrm{p}$ & $2 p$ & $2 p$ & $2 p$ & $2 \mathrm{p}$ \\
\hline basis & $1 e+2 e+3 e+3 e$ & $1 e+2 e+3 e+3 e$ & $1 e+2 e+3 e+3 e$ & $1 e+2 e+3 e+3 e$ & $1 e+2 e+3 e+3 e$ \\
\hline endopod & $3 e, 2 e, 2 e, 1 p+4 e$ & $3 e+1 p, 1 p, 0,2 e, 1 e+5 e$ & $3 \mathrm{e}+1 \mathrm{p}, 1 \mathrm{p}, 0,2 \mathrm{e}, 1 \mathrm{e}+5 \mathrm{e}$ & $3 \mathrm{e}+1 \mathrm{p}, 1 \mathrm{p}, 0,2 \mathrm{e}, 1 \mathrm{e}+5 \mathrm{e}$ & $3 \mathrm{e}+1 \mathrm{p}, 1 \mathrm{p}, 0,2 \mathrm{e}, 1 \mathrm{e}+5 \mathrm{e}$ \\
\hline exopod & $1 n+1 s+2 n$ & $1 n+1 s+4 n$ & $1 n+1 s+4 n$ & $2 n+4 n$ & $2 n+4 n$ \\
\hline \multicolumn{6}{|l|}{ Third maxilliped } \\
\hline coxa & 0 & 0 & 0 & 0 & le \\
\hline basis & $1 \mathrm{e}+1 \mathrm{e}+2 \mathrm{e}$ & $1 \mathrm{e}+1 \mathrm{e}+2 \mathrm{e}$ & $1 \mathrm{e}+1 \mathrm{e}+2 \mathrm{e}$ & $1 \mathrm{e}+1 \mathrm{e}+2 \mathrm{e}$ & $1 \mathrm{e}+1 \mathrm{e}+2 \mathrm{e}$ \\
\hline endopod & $2 \mathrm{e}, 0,2 \mathrm{e}, 1 \mathrm{e}+3 \mathrm{e}$ & $2 \mathrm{e}, 0,0,2 \mathrm{e}, 1 \mathrm{e}+3 \mathrm{e}$ & $2 \mathrm{e}, 0,0,2 \mathrm{e}, 1 \mathrm{e}+3 \mathrm{e}$ & $2 \mathrm{e}, 0,0,2 \mathrm{e}, 1 \mathrm{e}+3 \mathrm{e}$ & $2 \mathrm{e}, 0,0,3 \mathrm{e}, 1 \mathrm{e}+4 \mathrm{e}$ \\
\hline exopod & $2 n+3 n$ & $2 n+4 n$ & $2 n+4 n$ & $2 n+4 n$ & $2 n+4 n$ \\
\hline \multirow{5}{*}{$\begin{array}{l}\text { First pereiopod } \\
\text { coxa } \\
\text { basis } \\
\text { endopod } \\
\text { exopod }\end{array}$} & biramous bud & biramous bud & & & \\
\hline & & & 0 & 0 & 0 \\
\hline & & & $1 p+1 p+1 p$ & $1 p+1 p+1 p$ & $1 p+1 p+1 p$ \\
\hline & & & $2 \mathrm{e}, 1 \mathrm{e}, 0,2 \mathrm{e}, 1 \mathrm{e}+3 \mathrm{e}$ & $2 \mathrm{e}, 1 \mathrm{e}, 0,2 \mathrm{e}, 1 \mathrm{e}+4 \mathrm{e}$ & $2 \mathrm{e}, 1 \mathrm{e}, 1 \mathrm{e}, 3 \mathrm{e}, 4 \mathrm{e}+1 \mathrm{~d}$ \\
\hline & & & $2 n+4 n$ & $2 n+4 n$ & $2 n+4 n$ \\
\hline Second pereiopod & absent & absent & & & \\
\hline coxa & & & 0 & 0 & 0 \\
\hline basis & & & 0 & $1 \mathrm{e}+1 \mathrm{e}+2 \mathrm{e}$ & $1 e+1 e+2 e$ \\
\hline endopod & & & $2 \mathrm{e}, 1 \mathrm{~s}+3 \mathrm{e}$ & $2 \mathrm{e}, 0,0,2 \mathrm{e}, 1 \mathrm{~s}+3 \mathrm{e}$ & $2 \mathrm{e}, 1 \mathrm{e}, 1 \mathrm{e}, 2 \mathrm{e}, 2 \mathrm{e}+3 \mathrm{e}$ \\
\hline exopod & & & $2 n+4 n$ & $2 n+4 n$ & $2 n+4 n$ \\
\hline \multirow{5}{*}{$\begin{array}{l}\text { Third pereiopod } \\
\text { coxa } \\
\text { basis } \\
\text { endopod } \\
\text { exopod }\end{array}$} & absent & absent & absent & biramous bud & \\
\hline & & & & & 0 \\
\hline & & & & & le \\
\hline & & & & & $1 \mathrm{e}+1 \mathrm{e}+2 \mathrm{e}$ \\
\hline & & & & & $1 n+4 n$ \\
\hline Fourth pereiopod & absent & absent & absent & biramous bud & biramous bud \\
\hline Fifth pereiopod & absent & absent & absent & absent & absent \\
\hline \multicolumn{6}{|l|}{ Abdomen } \\
\hline somites & $0,0,0,0,2 \mathrm{~b}$ & $0,0,0,0,2 \mathrm{~b}$ & $0,0,0,0,2 \mathrm{~b}, 0$ & $0,0,0,0,2 \mathrm{~b}, 0$ & $0,0,0,0,2 \mathrm{~b}, 0$ \\
\hline Pleopods & absent & absent & absent & & absent \\
\hline \multirow{3}{*}{$\begin{array}{l}\text { Uropods } \\
\text { endopod } \\
\text { exopod }\end{array}$} & absent & absent & & & \\
\hline & & & $2 \mathrm{~s}$ & $6 \mathrm{p}$ & $10-12 p$ \\
\hline & & & $6 \mathrm{p}$ & $9 \mathrm{p}$ & $18-19 \mathrm{p}$ \\
\hline Telson & $7 d+7 d$ & $8 d+8 d$ & $(1 p+7 d)+(1 p+7 d)$ & $(1 p+5 d)+(1 p+5 d)$ & $(3 p+5 d)+(3 p+5 d)$ \\
\hline
\end{tabular}


Table 1 (Cont.). Setation and others characteristics of zoeal stages of Processa macrodactyla.

\begin{tabular}{|c|c|c|c|c|}
\hline \multirow[b]{2}{*}{ Features } & \multicolumn{4}{|c|}{ Stages } \\
\hline & Zoea VI & Zoea VII & Zoea VIII & Zoea IX \\
\hline Carapace length & $1.20 \pm 0.031$ & $1.51 \pm 0.017$ & $1.57 \pm 0.010$ & $1.64 \pm 0.008$ \\
\hline \multicolumn{5}{|l|}{ Antennule } \\
\hline peduncle & $1 c+19 p, 5 e+4 p$ & $1 c+24 p, 9 p$ & $1 c+24 p, 9 p$ & $1 c+27 p, 9 p$ \\
\hline endopod & $0,2 \mathrm{~s}+1 \mathrm{p}$ & $0,1 \mathrm{~s}, 2 \mathrm{~s}+1 \mathrm{p}$ & $0,3 \mathrm{~s}, 1 \mathrm{~s}, 5 \mathrm{e}$ & $1 \mathrm{e}, 6 \mathrm{e}, 2 \mathrm{e}, 5 \mathrm{e}$ \\
\hline exopod & $1 s+3 a$ & $3 \mathrm{e}, 3 \mathrm{e}, 3 \mathrm{e}$ & $3 p+4 p, 3 p, 3 e$ & $2 p+3 p+4 p+2 s, 5 p+2 s, 4 e$ \\
\hline \multicolumn{5}{|l|}{ Antenna } \\
\hline peduncle & 1c & $1 \mathrm{c}$ & $1 \mathrm{c}$ & $1 \mathrm{c}$ \\
\hline endopod & $0,5 \mathrm{~s}$ & 6-segmented & 8 -segmented & 8-segmented \\
\hline exopod & $17 p+1 j+3 p$ & $22 p+1 j+5 p$ & $22 p+1 j+5 p$ & $23 p+1 j+5 p$ \\
\hline \multicolumn{5}{|l|}{ Maxillule } \\
\hline coxal endite & $1 s+8 d$ & $2 \mathrm{e}+10 \mathrm{~d}$ & $2 \mathrm{e}+10 \mathrm{~d}$ & $2 \mathrm{e}+10 \mathrm{~d}$ \\
\hline basial endite & $1 s+12 t$ & $4 s+12 t$ & $4 s+12 t$ & $6 s+12 t$ \\
\hline endopod & $3 \mathrm{e}, 3 \mathrm{e}$ & $3 \mathrm{e}, 3 \mathrm{e}$ & $3 \mathrm{e}, 3 \mathrm{e}$ & $3 \mathrm{e}, 3 \mathrm{e}$ \\
\hline \multicolumn{5}{|l|}{ Maxilla } \\
\hline coxal endite & $(16 p)+(2 p+3 e)$ & $(16 p)+(2 p+3 e)$ & $(16 p)+(2 p+3 e)$ & $(16 p)+(2 p+3 e)$ \\
\hline basial endite & $(1 p+6 e)+(1 p+6 e)$ & $(1 p+6 e)+(1 p+6 e)$ & $(1 p+6 e)+(1 p+6 e)$ & $(1 p+6 e)+(1 p+6 e)$ \\
\hline endopod & $3 \mathrm{e}+2 \mathrm{e}, 1 \mathrm{e}+1 \mathrm{e}+2 \mathrm{e}$ & $3 \mathrm{e}+2 \mathrm{e}, 1 \mathrm{e}+1 \mathrm{e}+2 \mathrm{e}$ & $3 \mathrm{e}+2 \mathrm{e}, 1 \mathrm{e}+1 \mathrm{e}+2 \mathrm{e}$ & $3 \mathrm{e}+2 \mathrm{e}, 1 \mathrm{e}+1 \mathrm{e}+2 \mathrm{e}$ \\
\hline scaphognathite & $27 \mathrm{p}$ & $43 p$ & $43 p$ & $48 p$ \\
\hline \multicolumn{5}{|l|}{ First maxilliped } \\
\hline coxa & $10 \mathrm{p}$ & $10 p$ & $10 \mathrm{p}$ & $10 \mathrm{p}$ \\
\hline basis & $1 p+19 e$ & $1 p+19 e$ & $6 p+17 e$ & $26 \mathrm{e}$ \\
\hline endopod & $3 e+1 p, 1 e+1 p, 2 e, 1 e+3 e$ & $3 \mathrm{e}+1 \mathrm{p}, 1 \mathrm{e}+1 \mathrm{p}, 2 \mathrm{e}, 1 \mathrm{e}+3 \mathrm{e}$ & $3 e+1 p, 1 e+1 p, 2 e, 1 e+3 e$ & $3 e+1 p, 1 e+1 p, 2 e, 1 e+3 e$ \\
\hline exopod & $1 p+2 n+4 n$ & $1 p+2 n+4 n$ & $1 p+2 n+4 n$ & $1 p+2 n+4 n$ \\
\hline \multicolumn{5}{|l|}{ Second maxilliped } \\
\hline coxa & $2 \mathrm{p}$ & $2 p$ & $2 \mathrm{p}$ & $2 p$ \\
\hline basis & $1 e+2 e+3 e+3 e$ & $1 e+2 e+3 e+3 e$ & $1 e+2 e+3 e+3 e$ & $1 e+2 e+4 e+3 e$ \\
\hline endopod & $3 \mathrm{e}+1 \mathrm{p}, 2 \mathrm{e}+1 \mathrm{p}, 1 \mathrm{p}, 3 \mathrm{e}, 1 \mathrm{e}+6 \mathrm{e}$ & $3 e+1 p, 2 e+1 p, 1 p, 3 e, 6 e+1 d$ & $3 e+1 p, 2 e+1 p, 1 p, 4 e, 6 e+1 d$ & $3 \mathrm{e}+1 \mathrm{p}, 2 \mathrm{e}+1 \mathrm{p}, 1 \mathrm{p}, 4 \mathrm{e}, 6 \mathrm{e}+1 \mathrm{~d}$ \\
\hline exopod & $1 n+2 n+4 n$ & $2 n+2 n+4 n$ & $2 n+2 n+4 n$ & $2 n+2 n+4 n$ \\
\hline \multicolumn{5}{|l|}{ Third maxilliped } \\
\hline coxa & le & $1 \mathrm{e}$ & $1 \mathrm{e}$ & $1 \mathrm{e}$ \\
\hline basis & $1 e+1 e+2 e$ & $1 e+1 e+2 e$ & $1 e+1 e+2 e$ & $1 e+1 e+2 e$ \\
\hline endopod & $2 \mathrm{e}, 1 \mathrm{e}, 2 \mathrm{e}, 7 \mathrm{e}, 1 \mathrm{e}+4 \mathrm{e}$ & $2 \mathrm{e}, 1 \mathrm{e}+2 \mathrm{e}, 3 \mathrm{e}, 12 \mathrm{e}, 4 \mathrm{e}+1 \mathrm{~d}$ & $2 \mathrm{e}, 3 \mathrm{e}, 4 \mathrm{e}, 12 \mathrm{e}, 4 \mathrm{e}+1 \mathrm{~d}$ & $4 \mathrm{e}, 7 \mathrm{e}, 5 \mathrm{e}, 15 \mathrm{e}, 4 \mathrm{e}+1 \mathrm{~d}$ \\
\hline exopod & $2 n+2 n+4 n$ & $1 s+2 n+2 n+4 n$ & $1 s+2 n+2 n+4 n$ & $1 s+2 n+2 n+4 n$ \\
\hline \multicolumn{5}{|l|}{ First pereiopod } \\
\hline coxa & $1 \mathrm{p}$ & $1 \mathrm{p}$ & $1 \mathrm{p}$ & $1 \mathrm{p}$ \\
\hline basis & $1 p+1 p+1 p$ & $1 p+1 p+1 p$ & $1 p+1 p+1 p$ & $1 p+1 p+1 p$ \\
\hline endopod & $2 \mathrm{e}, 1 \mathrm{e}, 2 \mathrm{e}, 5 \mathrm{e}, 4 \mathrm{e}+1 \mathrm{~d}$ & $2 \mathrm{e}, 4 \mathrm{e}, 4 \mathrm{e}, 11 \mathrm{e}, 8 \mathrm{e}+1 \mathrm{~d}$ & $2 \mathrm{e}, 4 \mathrm{e}, 4 \mathrm{e}, 11 \mathrm{e}, 8 \mathrm{e}+1 \mathrm{~d}$ & $2 \mathrm{e}, 7 \mathrm{e}, 4 \mathrm{e}, 14 \mathrm{e}, 9 \mathrm{e}+1 \mathrm{~d}$ \\
\hline exopod & $2 n+2 n+4 n$ & $1 n+2 n+2 n+4 n$ & $2 n+2 n+2 n+4 n$ & $1-2 n+2 n+2 n+4 n$ \\
\hline \multicolumn{5}{|l|}{ Second pereiopod } \\
\hline coxa & 0 & 0 & 0 & 0 \\
\hline basis & $1 \mathrm{e}+1 \mathrm{e}+2 \mathrm{e}$ & $1 e+1 e+2 e$ & $1 \mathrm{e}+1 \mathrm{e}+2 \mathrm{e}$ & $1 e+1 e+2 e$ \\
\hline endopod & $2 \mathrm{e}, 1 \mathrm{e}, 1 \mathrm{e}, 4 \mathrm{e}, 5 \mathrm{e}+1 \mathrm{~d}$ & $3 e, 2 e, 3 e, 6 e, 6 e+1 d$ & $3 e, 2 e, 3 e, 6 e, 6 e+1 d$ & $3 e, 2 e, 3 e, 6 e, 6 e+1 d$ \\
\hline exopod & $2 n+2 n+4 n$ & $2 n+2 n+2 n+4 n$ & $2 n+2 n+2 n+4 n$ & $2 n+2 n+4 n$ \\
\hline \multicolumn{5}{|l|}{ Third pereiopod } \\
\hline coxa & 0 & 0 & 0 & 0 \\
\hline basis & $1 \mathrm{e}+1 \mathrm{e}+2 \mathrm{e}$ & $1 \mathrm{e}+1 \mathrm{e}+2 \mathrm{e}$ & $1 \mathrm{e}+1 \mathrm{e}+2 \mathrm{e}$ & $1 \mathrm{e}+1 \mathrm{e}+2 \mathrm{e}$ \\
\hline endopod & $2 \mathrm{e}, 2 \mathrm{e}, 2 \mathrm{e}, 5 \mathrm{e}, 3 \mathrm{e}+1 \mathrm{~d}$ & $4 \mathrm{e}, 4 \mathrm{e}, 4 \mathrm{e}, 12 \mathrm{e}, 7 \mathrm{e}+1 \mathrm{~d}$ & $4 \mathrm{e}, 4 \mathrm{e}, 4 \mathrm{e}, 12 \mathrm{e}, 7 \mathrm{e}+1 \mathrm{~d}$ & $5 e, 7 e, 7 e, 13 e, 7 e+1 d$ \\
\hline exopod & $1 n+2 n+4 n$ & $2 n+2 n+4 n$ & $2 n+2 n+4 n$ & $2 n+2 n+4 n$ \\
\hline \multicolumn{5}{|l|}{ Fourth pereiopod } \\
\hline coxa & 0 & 0 & 0 & 0 \\
\hline basis & $1 \mathrm{e}+1 \mathrm{e}$ & $1 \mathrm{e}+2 \mathrm{e}$ & $1 \mathrm{e}+1 \mathrm{e}+2 \mathrm{e}$ & $1 \mathrm{e}+1 \mathrm{e}+2 \mathrm{e}$ \\
\hline endopod & $2 \mathrm{e}, 1 \mathrm{e}, 2 \mathrm{e}, 5 \mathrm{e}, 2 \mathrm{e}+1 \mathrm{~d}$ & $3 \mathrm{e}, 4 \mathrm{e}, 4 \mathrm{e}, 8 \mathrm{e}, 7 \mathrm{e}+1 \mathrm{~d}$ & $4 \mathrm{e}, 5 \mathrm{e}, 6 \mathrm{e}, 12 \mathrm{e}, 7 \mathrm{e}+1 \mathrm{~d}$ & $4 \mathrm{e}, 7 \mathrm{e}, 8 \mathrm{e}, 16 \mathrm{e}, 7 \mathrm{e}+1 \mathrm{~d}$ \\
\hline exopod & $4 n$ & $2 n+4 n$ & $2 n+4 n$ & $2 n+4 n$ \\
\hline \multicolumn{5}{|l|}{ Fifth pereiopod } \\
\hline coxa & 0 & 0 & 0 & 0 \\
\hline basis & $1 \mathrm{e}+2 \mathrm{e}$ & $1 e+2 e$ & $1 \mathrm{e}+1 \mathrm{e}+2 \mathrm{e}$ & $1 e+1 e+2 e$ \\
\hline endopod & $2 \mathrm{e}, 1 \mathrm{e}, 1 \mathrm{e}, 4 \mathrm{e}, 1 \mathrm{e}+2 \mathrm{e}+1 \mathrm{~d}$ & $2 \mathrm{e}, 1 \mathrm{e}, 3 \mathrm{e}, 7 \mathrm{e}, 7 \mathrm{e}+1 \mathrm{~d}$ & $3 \mathrm{e}, 4 \mathrm{e}, 4 \mathrm{e}, 11 \mathrm{e}, 7 \mathrm{e}+1 \mathrm{~d}$ & $4 e, 8 e, 6 e, 16 e, 7 e+1 d$ \\
\hline Abdomen & & & & \\
\hline somites & $0,0,0,0,2 b, 1 b$ & $0,0,0,0,2 b, 1 b$ & $0,0,0,0,2 \mathrm{~b}, 1 \mathrm{~b}$ & $0,0,0,0,2 b, 1 b$ \\
\hline Pleopods & small buttons & biramous buds & biramous buds & biramous buds \\
\hline Uropods & & & & \\
\hline endopod & 17-18p & $46-48 p$ & $36-38 p$ & $31 p+13 e$ \\
\hline exopod & $26-28 p$ & $38-39 p$ & $36-39 p$ & $42 p+7 e$ \\
\hline Telson & $(3 s+5 d)+(3 s+5 d)$ & $(3 s+5 d)+(3 s+5 d)$ & $(3 s+5 d)+(3 s+5 d)$ & $(3 s+5 d)+(3 s+5 d)$ \\
\hline
\end{tabular}

a, aesthetasc; b, abdominal spine; c, spine; d, plumodenticulate seta; e, sparsely plumose seta; $\mathrm{j}$, spiny projection; n, plumose natatory seta; $p$, plumose seta; $s$, simple seta; $t$, plumodenticulate cuspidate setae. 
Table 2. Morphological differences in described larval stages of the European species of the genus Processa.

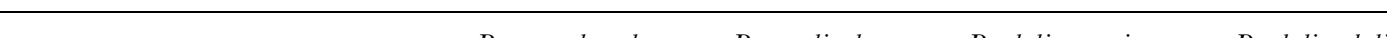

$\begin{array}{llll}\text { P. modica modica } & \text { P. modica carolii } & \text { P. nouveli holthuisi } & \text { P. nouveli nouveli }\end{array}$

\begin{tabular}{lcccc}
\hline Authors & Present study & $(1)$ & $(1),(2)$ & $(3),(4)$ \\
Carapace denticles & 4 & $5-8$ & $0-2$ & 4 \\
Length rostrum: frontal lobe ratio & $>1$ & $>1$ & $<1$ & $<1$ \\
Spine on stylocerite of & Present & Present $(3)$ & &
\end{tabular}

Spine on stylocerite of
antennula in ZVIII

Pterygostomian spine

Endopod of maxillule

Exopodal seta of maxillule

Endopod of maxilla

Median abdominal spines on

somite

Paired abdominal spines on

somite

No. of zoeal stages

Geographical distribution in

European waters*

Present
Marginal
$2,3(\mathrm{ZI})$
$1+2,3($ ZII - ZIX $)$
always present
$3+2+1+1+2$
$6($ from
ZVI $)$
5,6

$\begin{array}{cc}(5) & (3),(5) \\ 4 & 5 \\ =1 & =1\end{array}$

$(2),(5),(7)$
$3-4$
ZII-ZIII: $=$

(3), (7)

ZIV-ZIX: $>1$

$=1$

Present (3)

Present (3)

\section{Eastern Atlantic $\quad$ 8-9}

Eastern Atlantic

Mediterranean

Submarginal
2,3
ZI-ZIII
?
no
5

5

$\stackrel{8}{\text { Eastern }}$

Atlantic
Small

Long submarginal

$$
\text { 2, } 3
$$

ZI-ZIII
$2-4+2+1+1+2$

$2-4+2+1+1$
3

6 (from Z III)

5

7
Eastern

Eastern
Atlantic
Very long

Short almost marginal

Short

almost mar
$1+2,3$

$$
\begin{gathered}
\text { ZI-ZIII } \\
2+2+1+1+2 \\
\text { no }
\end{gathered}
$$

6 (from Z III)

5

Mediterranean

9
South-eastern

$$
\text { Spain }
$$

Spain

$$
5
$$

9

Eastern

Atlantic

?

?

no

\begin{tabular}{|c|c|c|c|c|c|c|c|c|}
\hline & $\begin{array}{l}\text { Processa } \\
\text { ?elegantula }\end{array}$ & $\begin{array}{l}\text { Processa } \\
\text { sp.1 }\end{array}$ & $\begin{array}{l}\text { Processa } \\
\text { sp. } 2\end{array}$ & $\begin{array}{l}\text { Processa } \\
\text { EM4 }\end{array}$ & $\begin{array}{l}\text { Processa } \\
\text { EM5 }\end{array}$ & $\begin{array}{l}\text { Processa } \\
\text { EM6 }\end{array}$ & $\begin{array}{l}\text { Processa } \\
\text { EM7 }\end{array}$ & $\begin{array}{l}\text { Processa } \\
\text { EFSL11 }\end{array}$ \\
\hline Authors & $(3)$ & $(3)$ & $(3)$ & (9) & $(7),(9)$ & $(7),(9)$ & (9) & $(7)$ \\
\hline Carapace denticles & 5 & $3-4$ & $6-7$ & 0 & 4 & 9 & $3-6$ & $5-6$ \\
\hline Length rostrum: frontal lobe ratio & $>1$ & & $>1$ & & $=1$ & $>1$ & & $=1$ \\
\hline $\begin{array}{l}\text { Spine on stylocerite of } \\
\text { antennula in ZVIII }\end{array}$ & Present (3) & No $(3)$ & No $(3)$ & & & & & \\
\hline Pterygostomian spine & Long & Short & Long & $\begin{array}{c}\text { Slightly } \\
\text { submarginal }\end{array}$ & $\begin{array}{c}\text { Markedly } \\
\text { submarginal }\end{array}$ & Almost marginal & $\begin{array}{l}\text { Marginal or } \\
\text { almost marginal }\end{array}$ & Long \\
\hline Endopod of maxillule & ? & ? & ? & ? & $?^{\circ}$ & ? & ? & ? \\
\hline Exopodal seta of maxillule & ? & ? & ? & ? & ? & ? & ? & ? \\
\hline Endopod of maxilla & ? & ? & ? & ? & ? & ? & ? & ? \\
\hline $\begin{array}{l}\text { Median abdominal spines on } \\
\text { somite }\end{array}$ & no & no & no & $?$ & no & no & no & no \\
\hline $\begin{array}{l}\text { Paired abdominal spines on } \\
\text { somite }\end{array}$ & 5 & 5 & 5 & 5 & 5 & 5 & 5 & 5 \\
\hline No. of zoeal stages & 8 & 8 & 8 & $?$ & 9 & 9 & 9 & 9 \\
\hline $\begin{array}{l}\text { Geographical distribution in } \\
\text { European waters** }\end{array}$ & $\begin{array}{l}\text { Mediterranean } \\
\text { French coast }\end{array}$ & $\begin{array}{l}\text { Mediterranean } \\
\text { French coast }\end{array}$ & $\begin{array}{l}\text { Mediterranean } \\
\text { French coast }\end{array}$ & Israel coast & $\begin{array}{c}\text { Israel coast } \\
\text { Portuguese coast }\end{array}$ & $\begin{array}{l}\text { Israel coast } \\
\text { Portuguese coast }\end{array}$ & Israel coast & Portuguese coast \\
\hline
\end{tabular}

5

9

Mediterranean

$+2$

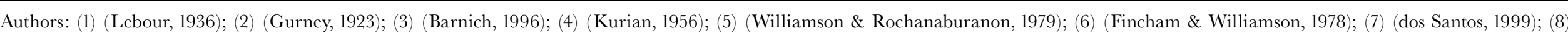

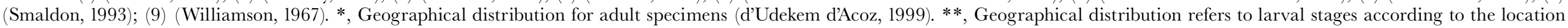
where collected. 
Table 3. Time of appearance of pereiopods and phase of development in described zoeal stages of genus Processa.

\begin{tabular}{|c|c|c|c|c|c|}
\hline & $\begin{array}{l}\text { P. macrodactyla } \\
\text { (Present study) }\end{array}$ & $\begin{array}{c}\text { P. modica modica } \\
\text { (Williamson \& } \\
\text { Rochanaburanon, 1979) }\end{array}$ & $\begin{array}{l}\text { P. canaliculata } \\
\text { (Lebour, 1936) }\end{array}$ & $\begin{array}{l}\text { P. edulis crassipes } \\
\text { (Gurney, 1923) } \\
\text { (Lebour, 1936) }\end{array}$ & $\begin{array}{c}\text { P. nouveli holthuisi } \\
\text { (Williamson \& } \\
\text { Rochanaburanon, 1979) }\end{array}$ \\
\hline Zoea I & $1 \mathrm{P}$ bud & $1 \mathrm{P}$ bud & ? & $1 \mathrm{P}$ bud & ? \\
\hline Zoea II & $1 \mathrm{P}$ bud & $\begin{array}{l}1 \mathrm{P} \text { fuctional } \\
2 \mathrm{P} \text { bud } \\
3 \mathrm{P} \text { bud } \\
4 \mathrm{P} \text { bud } \\
5 \mathrm{P} \text { bud }\end{array}$ & $1 \mathrm{P}$ functional & $\begin{array}{l}1 \mathrm{P} \text { functional } \\
2 \mathrm{P} \text { bud } \\
3 \mathrm{P} \text { bud } \\
4 \mathrm{P} \text { bud } \\
5 \mathrm{P} \text { bud }\end{array}$ & 1P functional \\
\hline Zoea III & $\begin{array}{l}1 \mathrm{P} \text { functional } \\
2 \mathrm{P} \text { functional }\end{array}$ & $\begin{array}{l}1 \mathrm{P} \text { functional } \\
2 \mathrm{P} \text { bud } \\
3 \mathrm{P} \text { bud } \\
4 \mathrm{P} \text { bud } \\
5 \mathrm{P} \text { bud }\end{array}$ & $\begin{array}{l}1 \mathrm{P} \text { functional } \\
2 \mathrm{P} \text { functional }\end{array}$ & $\begin{array}{l}1 \mathrm{P} \text { functional } \\
2 \mathrm{P} \text { functional } \\
3 \mathrm{P} \text { bud } \\
4 \mathrm{P} \text { bud } \\
5 \mathrm{P} \text { bud }\end{array}$ & $\begin{array}{l}1 \mathrm{P} \text { functional } \\
2 \mathrm{P} \text { functional }\end{array}$ \\
\hline Zoea IV & $\begin{array}{l}\text { 1P functional } \\
2 \mathrm{P} \text { functional } \\
3 \mathrm{P} \text { bud } \\
4 \mathrm{P} \text { bud }\end{array}$ & $\begin{array}{l}1 \mathrm{P} \text { functional } \\
2 \mathrm{P} \text { functional } \\
3 \mathrm{P} \text { bud } \\
4 \mathrm{P} \text { bud } \\
5 \mathrm{P} \text { bud }\end{array}$ & $\begin{array}{l}\text { 1P functional } \\
\text { 2P functional } \\
\text { 3P functional }\end{array}$ & $\begin{array}{l}\text { 1P functional } \\
2 \mathrm{P} \text { functional } \\
3 \mathrm{P} \text { functional } \\
4 \mathrm{P} \text { bud } \\
5 \mathrm{P} \text { bud }\end{array}$ & $\begin{array}{l}1 \mathrm{P} \text { functional } \\
2 \mathrm{P} \text { functional } \\
3 \mathrm{P} \text { functional } \\
4 \mathrm{P} \text { functional? } \\
5 \mathrm{P} \text { functional? }\end{array}$ \\
\hline Zoea V & $\begin{array}{l}\text { 1P functional } \\
2 \mathrm{P} \text { functional } \\
3 \mathrm{P} \text { functional } \\
\quad 4 \mathrm{P} \text { bud }\end{array}$ & $\begin{array}{l}1 \mathrm{P} \text { functional } \\
2 \mathrm{P} \text { functional } \\
3 \mathrm{P} \text { functional } \\
4 \mathrm{P} \text { bud } \\
5 \mathrm{P} \text { bud }\end{array}$ & $\begin{array}{l}1 \mathrm{P} \text { functional } \\
2 \mathrm{P} \text { functional } \\
3 \mathrm{P} \text { functional } \\
4 \mathrm{P} \text { functional } \\
5 \mathrm{P} \text { functional? }\end{array}$ & $\begin{array}{l}\text { 1P functional } \\
2 \mathrm{P} \text { functional } \\
3 \mathrm{P} \text { functional } \\
4 \mathrm{P} \text { functional } \\
5 \mathrm{P} \text { bud }\end{array}$ & $\begin{array}{l}1 \mathrm{P} \text { functional } \\
2 \mathrm{P} \text { functional } \\
3 \mathrm{P} \text { functional } \\
4 \mathrm{P} \text { functional } \\
5 \mathrm{P} \text { functional? }\end{array}$ \\
\hline Zoea VI-IX & $\begin{array}{l}\text { 1P functional } \\
\text { 2P functional } \\
\text { 3P functional } \\
\text { 4P functional } \\
\text { 5P functional }\end{array}$ & $\begin{array}{l}\text { 1P functional } \\
2 \mathrm{P} \text { functional } \\
3 \mathrm{P} \text { functional } \\
4 \mathrm{P} \text { functional } \\
5 \mathrm{P} \text { functional }\end{array}$ & $\begin{array}{l}1 \mathrm{P} \text { functional } \\
2 \mathrm{P} \text { functional } \\
3 \mathrm{P} \text { functional } \\
4 \mathrm{P} \text { functional } \\
5 \mathrm{P} \text { functional }\end{array}$ & $\begin{array}{l}\text { 1P functional } \\
2 \mathrm{P} \text { functional } \\
3 \mathrm{P} \text { functional } \\
4 \mathrm{P} \text { functional } \\
5 \mathrm{P} \text { functional }\end{array}$ & $\begin{array}{l}\text { 1P functional } \\
2 \mathrm{P} \text { functional } \\
3 \mathrm{P} \text { functional } \\
4 \mathrm{P} \text { functional } \\
5 \mathrm{P} \text { functional }\end{array}$ \\
\hline
\end{tabular}

(1), there are no ZVIII and ZIX zoeal stages for P. modica; neither ZIX zoeal stage for P. edulis.

containing well-aerated filtered natural seawater (36 salinity) until hatching. No food was added. Females released larvae $48 \mathrm{~h}$ and $72 \mathrm{~h}$ after their collection in a total amount of approximately 2000 and 500 larvae, respectively. After hatching, actively swimming larvae were transferred to 1-l glass bottles with aeration at constant temperature $\left(22^{\circ} \mathrm{C} \pm 1\right)$ and fed with Artemia nauplii. The water was changed daily, and larvae were checked for evidence of moulting. Each time the water was renewed, 3-4 larvae were preserved in $4 \%$ formalin. Rearing was terminated when larvae moulted to the first juvenile instar.

Descriptions of different instars were based on at least ten specimens of each larva. The appendages were dissected in water, mounted in Faure's liquid and drawn using an interference phase microscope with camera lucida. General recommendations proposed by Clark et al. (1998) for standardization in larval descriptions were followed. Carapace length (CL) was measured from the anterior margin of eyes to the posterior carapace margin. The sizes given are the arithmetic mean $\pm 95 \%$ confidence intervals. Other drawings of different parts of larval stages are available. Contact authors if needed.

\section{RESULTS}

The complete planktonic development of Processa macrodactyla took place through nine zoeal stages. At $22^{\circ} \mathrm{C} \pm 1$ and 36 salinity the juvenile stage appeared 23 days after hatching. The major features of each larval stage and changes in appendage setation follow. Details of the type and distribution of setae and other features are given in Table 1. Table 2 shows the main morphological differences between described larvae of the genus Processa and those of which only partial descriptions have been made without assigning a certain species. Table 3 shows the time of appearance of pereiopods and the presence of exopods. The first larval stage of Processa macrodactyla previously described by González-Gordillo \& Rodríguez (2000) is added here to make comparison easier.

\section{Processa macrodactyla Holthuis, 1952}

(Figures 1-3, Tables 1-3)

Previous description: Zoea I (González-Gordillo \& Rodríguez, 2000: 95, figures 3-4, table 1)

\section{General morphology of zoeal stages}

Carapace (Figures 1A,C,D \& 2D). Flattened, with pterygostomian spine present and four small teeth on anteroventral margin. Mediodorsal tubercle on anterior part of carapace in first two stages and on anterior and posterior parts of carapace in subsequent stages. Rostrum absent in first stage but this and supraorbital spine appearing from second stage on. Eyes stalked from second larval stage.

Antennule (Figure 3F,I): slender with unsegmented peduncle in two first stages. Endopod as a long plumose seta.

Antenna (Figure 3G,H): endopod unsegmented, elongated and tapering spine process bearing a row of minute 


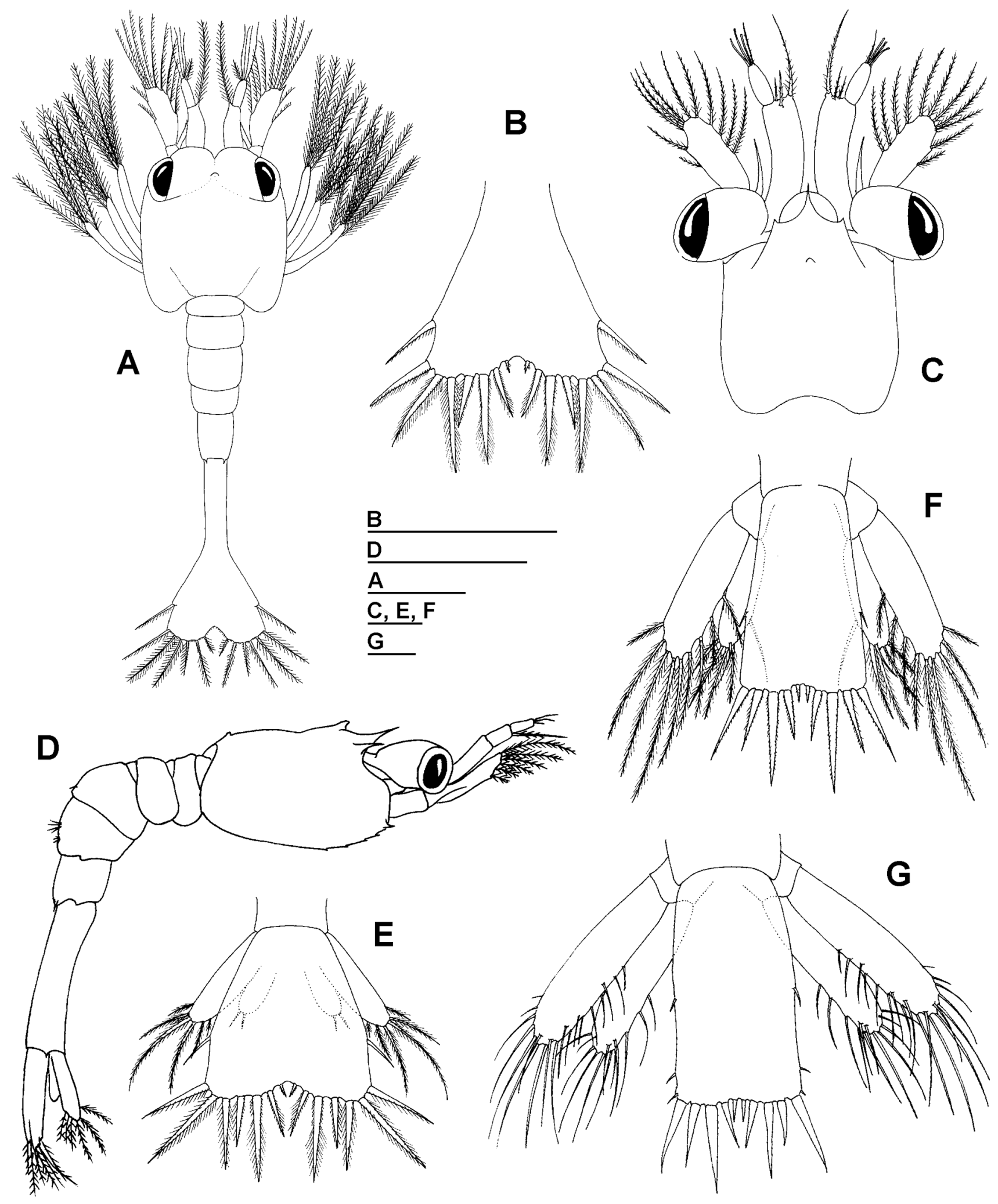

Figure 1. Processa macrodactyla. (A) Zoea I: dorsal view; (B-C) Zoea II: (B) telson; (C) cephalothorax dorsal view; (D-E) Zoea III: (D) lateral view; (E) uropods and telson; (F) Zoea IV: uropods and telson; (G) Zoea V: uropods and telson. Scale bars: A, $250 \mu \mathrm{m} ; \mathrm{B}, \mathrm{D}, 500 \mu \mathrm{m} ; \mathrm{C}, \mathrm{E}-\mathrm{G}, 100 \mu \mathrm{m}$. 


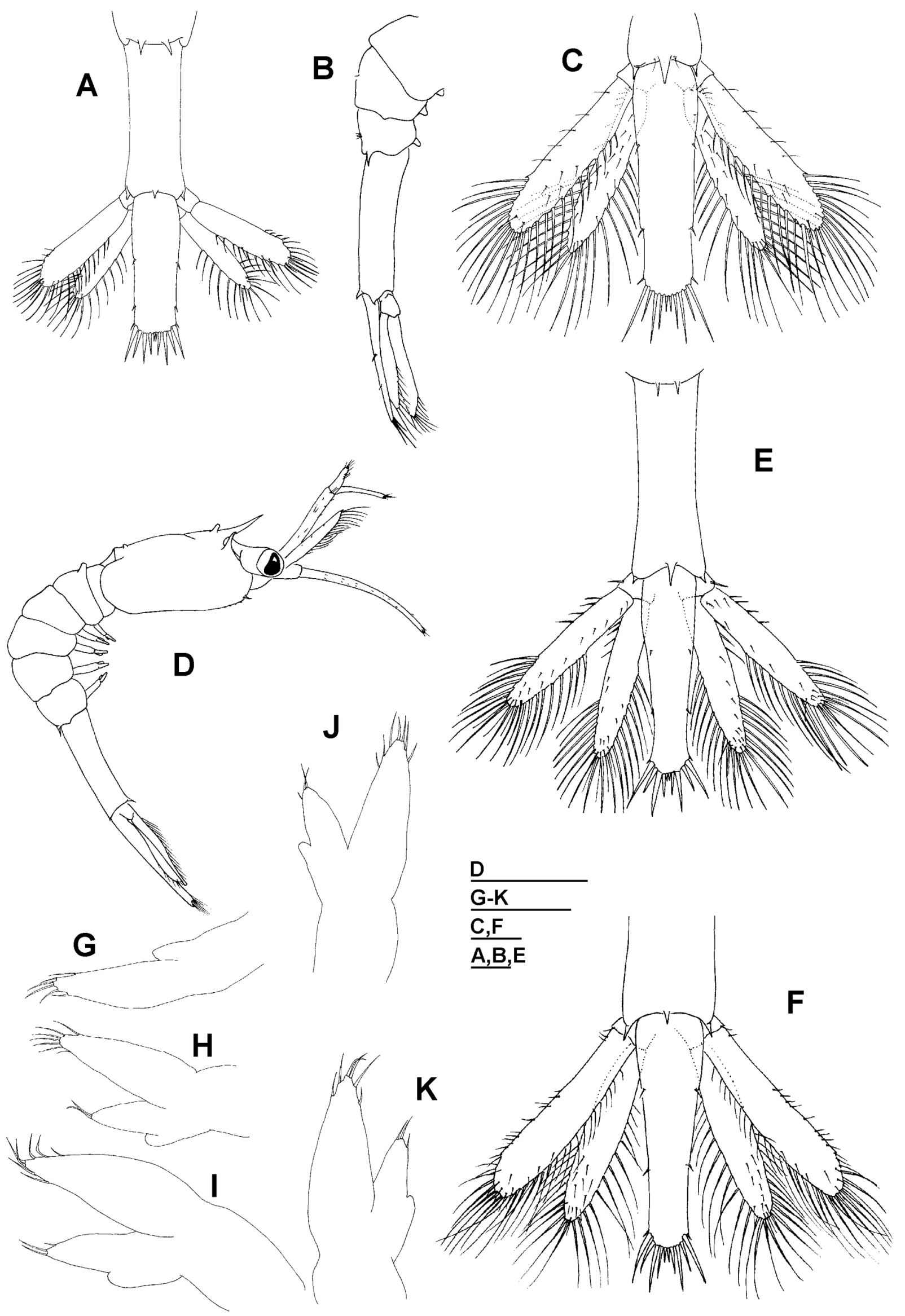

Figure 2. Processa macrodactyla. (A-B) Zoea VI: (A) uropods and telson; (B) lateral view of abdomen; (G) Zoea VII: uropods and telson; (D-E) Zoea VIII: (D) lateral view; (E) uropods and telson; (F-K) Zoea IX: (F) uropods and telson; (G) first pleopod; $(\mathrm{H})$ second pleopod; (I) third pleopod; (J) fourth pleopod; (K) fifth pleopod. Scale bars: A-C, E-K, $200 \mu \mathrm{m} ; \mathrm{D}, 1 \mathrm{~mm}$. 

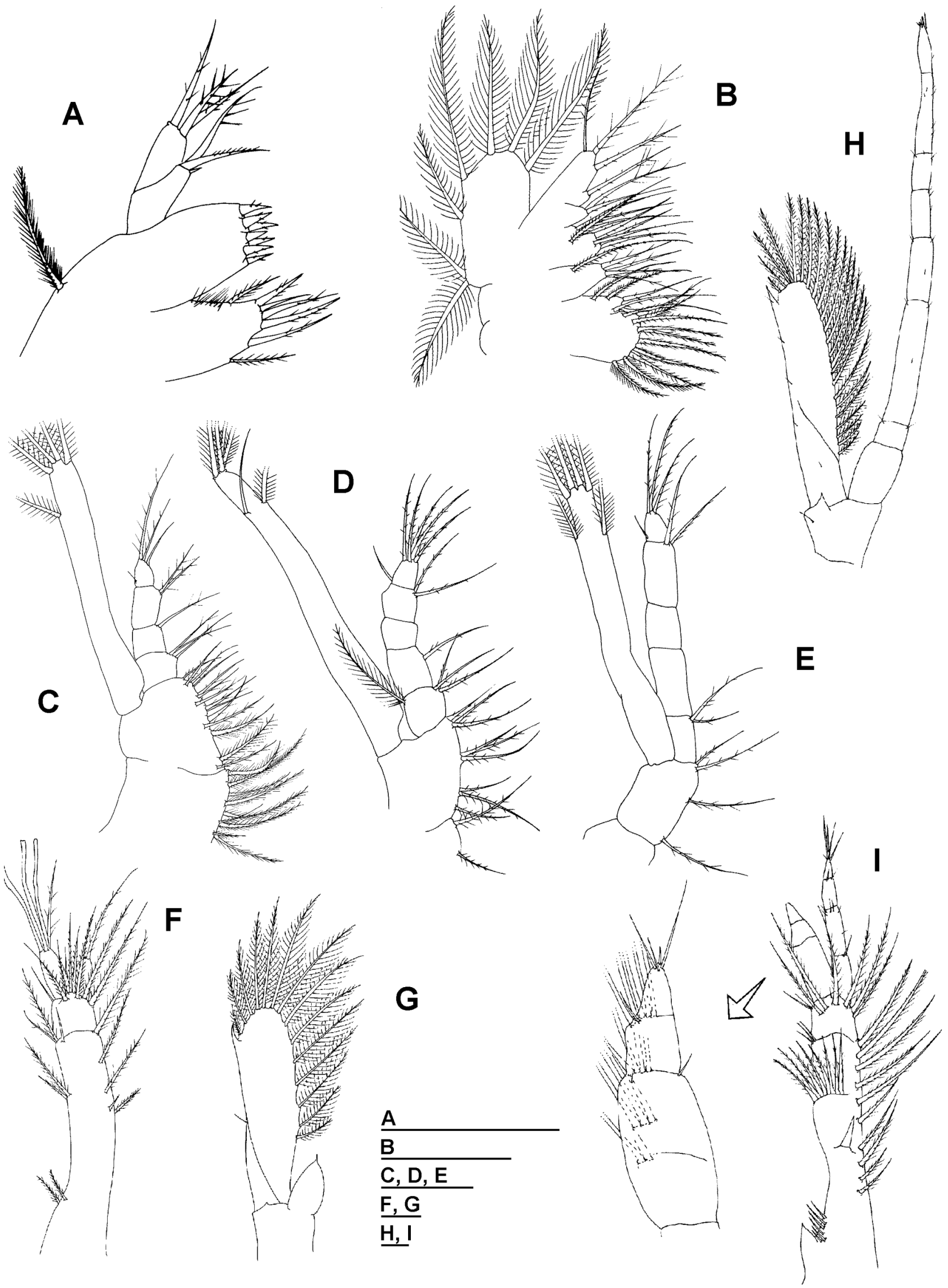

Figure 3. Processa macrodactyla. (A-E) Zoea II: (A) maxillule; (B) maxilla; (G) first maxilliped; (D) second maxilliped; (E) third maxilliped; (F-G) Zoea V: (F) antennule; (G) antenna; (H-I) Zoea IX: (H) antenna; (I) antennule. Scale bars: $100 \mu \mathrm{m}$. 


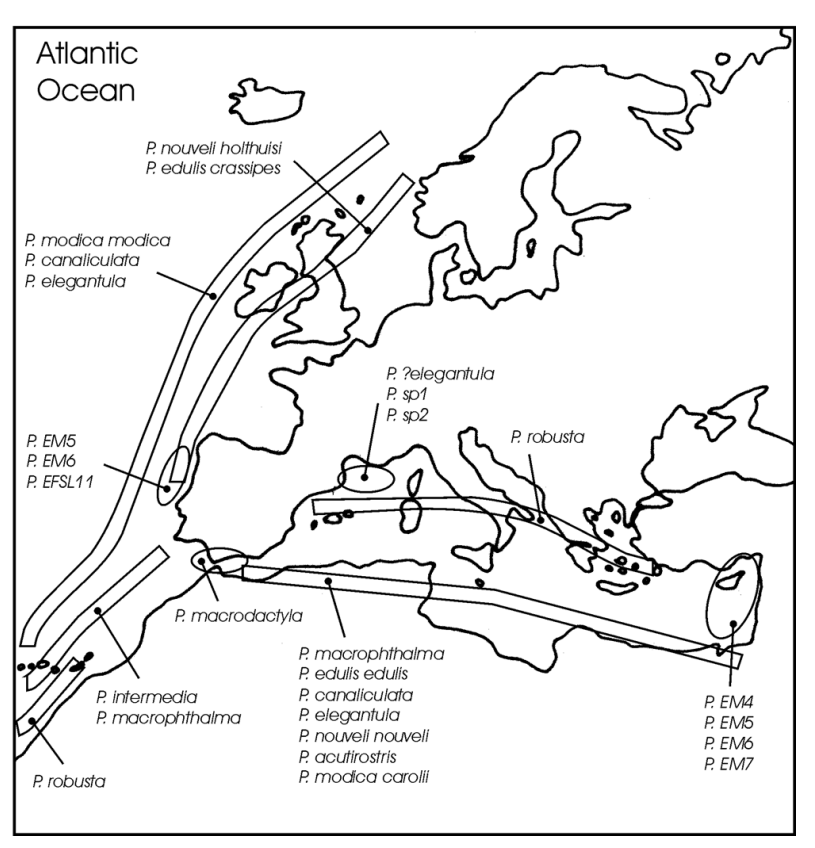

Figure 4. European distribution of recorded Processa species.

spines in first five stages, and not extending beyond middle of exopod. In subsequent stages the endopod is segmented and exceeds middle of exopod. Exopod unsegmented and broad.

Mandible: palp absent.

Maxillule (Figure 3A): endopod 2-segmented with number of setae unchanged from second zoeal stages. Exopod present as a long plumose seta.

Maxilla (Figure 3B): coxal and basial endites bilobed. Endopod unsegmented and tetralobed, setation unchanged.

First maxilliped (Figure 3G): endopod 4-segmented extending beyond middle of exopod. Exopod unsegmented.

Second maxilliped (Figure 3D): endopod 4-segmented, extending beyond middle of exopod. Exopod unsegmented.

Third maxilliped (Figure 3E): coxa naked. Endopod 4-segmented as long as exopod in four first stages and exceeding the length of exopod in subsequent stages. Exopod unsegmented.

Pereiopods: progressive development throughout zoeal stages. First to fifth pereiopods fully functional from sixth stage. First to fourth pereiopods biramous, fifth pereiopod uniramous. First and second right pereiopods with cheliped-like form from seventh zoeal stage.

Abdomen (Figures 1A,D \& 2B,D): five somites and telson in two first stages, sixth additional somite in subsequent stages. Somite 4 bears a dorsal tuft of short setae. Somite 5 with a pair of small dorsal spines.

Pleopods (Figure 2G-K): present as small buttons in sixth larval stage; biramous buds in seventh stage.

Uropods (Figures 1E,F,G \& 2A, C,E,F): present from third zoeal stage.

Telson (Figures 1B,E,G \& 2A, G,E,F): broad posteriorly, with a median cleft in four first stages and almost rectangular shaped in subsequent stages. Tapering towards posterior end in last stage. Central pair of setae smallest.

\section{DISCUSSION}

The larval morphology of the studied species Processa macrodactyla is a typical example of larvae belonging to the Infraorder Caridea, presenting carapace laterally flattened and telson flattened with usually seven (Zoea I) or eight (later zoeae) setae on each half margin, and without median spine (dos Santos \& González-Gordillo, 2004). Other characteristic features can also be useful to distinguish between families. Larvae of the family Processidae have been compared with those of Pandalidae, Hippolytidae and Crangonidae as they seem to be closer to each other than to other families. In Pandalidae, as in Processidae, the distance between bases of antennules is higher than the width of each antennule, but the presence of an unsegmented antennal exopod and an untoothedrostrum are distinctive characters of processid larvae. Hippolytidae larvae differ from Processidae larvae in two main characters: (1) the distance between bases of antennules is lower than the width of each antennule; and (2) the presence of an anal spine since the first stage of development. Crangonidae shares with Processidae an unsegmented antennal exopod and the presence of a subchelate first pereiopod in later stages. However, they differ in the form of the endopod of antennule that is long and thin in Processidae, and in the absence of supraorbital spines in all larval stages of Crangonidae. In addition to the differences in the larval morphology, the long series of development and its structure in Processidae is completely different from that shown by Crangonidae.

The larval stages of $P$. macrodactyla share with its species congeners the following characters: the dorso-lateral spines on the fifth abdominal somite (Lebour, 1936); the absence of rostrum in Zoea I and its short-size presence in later stages; the presence of an anal spine in later zoeal stages (Williamson \& Rochanaburanon, 1979); the presence of a pair of supraorbital spines from the second zoeal stage on; the presence of a pterygostomian spine (Williamson \& Rochanaburanon, 1979); the presence of functional maxillipeds from Stage I on (dos Santos, 1999); when present, the fifth pereiopod has the same size as the other pereiopods and lacks exopod; and finally, telson without a deep central invagination (dos Santos \& González-Gordillo, 2004). In contrast, other features that remain unchanged throughout the development of P. macrodactyla, such as the number of denticles on the ventral carapace margin, the presence of exopodal seta in the maxillule, and the setation of the endopod of the maxillule and maxilla, can be used to distinguish it from other congeneric species (Table 2).

The number of carapace denticles is a common feature often used by other authors to distinguish between the larvae of the species of the genus Processa. It presents 4 in P. macrodactyla and 5-8 in $P$. canaliculata. It also differs between subspecies: it is $0-2$ in $P$. edulis crassipes while 4 in $P$. edulis edulis and 5 in $P$. modica carolii but 4 in $P$. modica modica. It was said to be 3 in $P$. nouveli holthuisi (Williamson \& Rochanaburanon, 1979) and 3 or 4 in P. nouveli nouveli (Barnich, 1996), but dos Santos, 1999 noted 3 or 4 for the first subspecies and 3-6 for the second one. With regard to what has been previously stated, specimens with more than four carapace denticles were considered to be $P$. nouveli nouveli in such a way that this species could have 
been overestimated while $P$. nouveli holthuisi could have been underestimated (dos Santos, 1999). For this reason, when referrring to carapace denticles it is important to take into account the geographical distribution (see Figure 4), the place where samples come from and larval size in each stage.

The length or location of pterygostomian spine and the ratio between rostrum length and frontal lobe have also been used by some authors in order to try to clarify larval identification, as they are features that remain invariable, or almost invariable, along the larval development. Also the presence or absence of a spine on the stylocerite of the antennule has been used to identify between adult species of Processa (Smaldon, 1993) as well as some undetermined Processa species. The setation of the endopod of maxillule in the first stage of $P$. macrodactyla is 2,3 , as in $P$. modica modica and $P$. edulis crassipes (not described in the text of Gurney, 1923 but drawn) and it changes for the successive stages to $1+2,3$, as in $P$. nouveli holthuisi. The exopodal seta of maxillule is always present in $P$. macrodactyla but it is only observed in the three first zoeal stages of $P$. modica modica, $P$. edulis crassipes and $P$. nouveli holthuisi. The 5-lobed endopod of maxilla only shows differences between species in the setation of the proximal lobe, bearing two setae in $P$. nouveli holthuisi, three setae in $P$. macrodactyla and 2-4 setae in $P$. modica modica. The remaining features of the appendages shown in $P$. macrodactyla, as the setation of the coxa and the basis of maxillipeds and pereiopods, cannot be compared with those from the other species mentioned because no descriptions are available.

Known morphological differences between larval stages of different Processa are also based on the presence or absence of abdominal spines. All of the described species have a pair of dorso-lateral spines on somite $\mathrm{V}$, which is a common characteristic in this genus. Processa canaliculata has another pair of dorso-lateral spines on the fourth abdominal segment which may be much reduced but is always present (Lebour, 1936). Processa modica is the only species compared presenting a small median dorsal spine on abdominal somite 3 and another one on somite 6 , similar in length to those on somite 5, present from Stage III (Williamson \& Rochanaburanon, 1979), which definitely characterizes this species. It is peculiar of P. macrodactyla also to show a median abdominal spine on somite 6 from Zoea VI.

Relative to the total zoeal number of the larval series nine stages are described for $P$. modica carolii. In previous descriptions seven zoeal stages were assigned to $P$. modica modica showing this as a distinguishing feature between $P$. modica subspecies since they are not always morphologically distinct (Williamson \& Rochanaburanon, 1979). The zoeal stages are eight or nine for $P$. edulis and $P$. canaliculata, while nine for $P$. nouveli. In the present study, also nine zoeal stages were found for P. macrodactyla. As mentioned by Lebour (1936) the larvae of $P$. canaliculata and $P$. edulis crassipes from the inshore plankton moulted to the decapodit stage after ZVIII, whereas larvae from the offshore plankton moulted only after ZIX to the decapodit so as to improve their chances of reaching the coast again. Barnich (1996), points out that this could be the reason why ZIX stages of Processa can miss when taken from coastal plankton samples. According to Lebour, the postlarvae reared from ZVIII stages are smaller and less developed than those from ZIX stages (Barnich, 1996). Also Gurney (1923) pointed out the same, as well as the fact that one stage may represent more than one moult. In this way, it can be observed from the results obtained for $P$. macrodactyla, an outstanding change in the setation between an early zoeal stage group (ZI to ZIV-V) and a later zoeal stage group ( $\mathrm{ZV}-\mathrm{VI}$ to $\mathrm{ZIX})$. The main changes in the setation are referred to the endopod and exopod of the antennule, peduncle and endopod of the antenna, coxal endite of the maxillule, coxal and basial endites of the maxilla, coxa and endopod of the first maxilliped, endopod of the second maxilliped, coxa, endopod and exopod of the third maxilliped, coxa of the first pereiopod, endopod and exopod of the second pereiopod, basis of the third pereiopod, pleopods and telson. These characters remain, with few exceptions, invariable from ZI to ZIV or ZV, to then change and maintain until the last zoeal stage. It has not been noticed in other species of the genus so it might not exist, or otherwise it might have been omitted owing to incomplete descriptions. Some characters of Zoea V are shared with previous stages, while others are found in more developed stages. This is in accordance with a combinatorial moulting situation, where morphological mechanisms are accelerated or retarded resulting in an instar exhibiting heterochrony in its anatomical features. Similarly, Gurney (1923) describing P. nouveli holthuisi pointed out that there is a considerable variation among the specimens of this ZV stage and there might be in some cases, intermediate moults during Stage $\mathrm{V}$ with the result that some larvae unite the characters of Stages ZIV and ZV, and others those of ZV and ZVI.

There are a few larval forms of Processa which some authors could not ascertain to a species level. Williamson (1967) described some larvae from the eastern Mediterranean. One of them is Processa EM4 which, according to the number of carapace denticles and the location of pterygostomian spine, is tempting to ascribe to P. edulis crassipes. However, the geographical distribution rejects this possibility as Nouvel \& Holthuis (1957) mentioned this subspecies to appear only in Atlantic waters (Figure 4). On the other hand, Barnich (1996) also referred to these Processa EM4 when citing new larvae called Processa sp.l collected along French Mediterranean coasts. These are found to be very similar to Processa EM4 with the only obvious difference being the absence of carapace denticles in Processa EM4 and the presence of 3-4 denticles in Processa sp.1. However, we consider this a relevant feature in a way that Processa sp.l can be considered more alike, between known larval species, to Processa edulis edulis. Nevertheless, the description given is insufficient to certainly designate a known species larvae. Barnich (1996) also described Processa sp.2, with no matching descriptions between these and known Processa larvae. Even though the larvae of $P$. robusta, $P$. acutirostris, $P$. elegantula and $P$. macrophthalma are unknown up to now their adults occur in the same area where both Processa sp.1 and Processa sp.2 were sampled. Williamson (1967) also mentioned larvae he called Processa EM5, giving morphological features but being unable to find resemblances with other larval species. Later, dos Santos (1999) collected these larvae in the Portuguese coasts (North Atlantic). dos Santos (1999) considered the possibility of 
ascribing this Processa EM5 to P. macrophthalma as this species was already cited along Portuguese coasts by Neves (1973) and the adults are found to live in both areas where larvae were collected. Another partial description made by Williamson (1967) is Processa EM6 and it was reviewed by dos Santos (1999). dos Santos (1999) mentioned $P$. intermedia as the only unknown larvae to occur in Portuguese coasts. But the adult stage of this species has not been found in the eastern Mediterranean (d'Udekem d'Acoz, 1999) where Processa EM6 was first sampled. Otherwise, P. elegantula is distributed both along the Atlantic coasts and in the Mediterranean. Hence, according to Barnich (1996) Processa EM6 could be ascribed as $P$. elegantula despite some morphological differences. Williamson (1967) ascribed the Processa EM7 larvae observed in eastern Mediterranean to $P$. canaliculata after Bourdillon-Casanova (1960), observations supported by the high abundance of this species in the area. Still, no additional pair of spines on somite 4 is punctuated on Processa EM7 and the given size of the last zoea differs greatly between the two species. Barnich (1996) corresponded Processa EM7 with $P$. nouveli nouveli, with which it is more alike. Later, dos Santos (1999) found in Portuguese waters larvae similar to those of Processa EM7, and these were also referred to as $P$. nouveli nouveli (A. dos Santos, personal communication). Finally, dos Santos (1999) collected in the same area larvae of Processa named as Processa EFSL11 which was not ascribed to any species with larval development known along Portuguese coasts or European waters. Nevertheless, $P$. intermedia, with unknown larval development, included in its distribution the south coasts of Portugal, being able to correspond the larvae of Processa EFSL 11 to those of P. intermedia. Yet, the possibility that more unknown Processa species can be recorded is something to bear in mind (dos Santos, 1999).

The time of appearance of the pereiopods in the larval development and the presence of exopods are also important taxonomic characters to identify shrimp larvae. In Table 3 the presence of pereiopods and phase of development are listed (bud/functional) in each larval stage of those Processa species where they are described. With regard to this feature, $P$. macrodactyla and $P$. modica modica are the species with the slowest pereiopodal development while the fastest are $P$. canaliculata, $P$. edulis crassipes and P. nouveli holthuisi. In P. macrodactyla, the first pereiopod is functional only from Zoea III on, at the same time as the second one, while in the other species the first pereiopod is functional from Zoea II. The third pereiopod is only functional from Zoea V on in P. macrodactyla and P. modica modica while $P$. canaliculata, $P$. edulis crassipes and $P$. nouveli holthuisi already show a functional fourth pereiopod. The full development of the pereiopods in the studied processid species is achieved in Zoea VI. Taking this into account, we can easily distinguish the five early larval stages of Processa from the later stages and even differentiate between species of the genus. On the other hand, $P$. modica modica and $P$. edulis crassipes are the species with shorter larval series (usually seven and eight zoeal stages, respectively) and it is precisely these species that show all pereiopods (as buds, mainly) from Zoea II on. In contrast, the full appearance of the pereiopods (including buds) in the other species is only observed from Zoea $\mathrm{V}$ on. It is possible that the reduction of the number of zoeal stages in the larval development involves a premature appearance of all pereiopods at once. Gurney (1942) also pointed out that the abbreviation of the larval life tends to the suppression of the exopods on the pereiopods. This feature is observed in $P$. modica modica, which is the unique species without exopods on the fourth pereiopod.

It appears to be typical of the Caridea type of development that: (1) their moults are gradual, often associated with little or no change in size, morphology, and biomass; and (2) both the number of instars within a larval phase and the morphological characters of an instar vary intraspecifically (Anger, 2001). This is what makes it difficult to discover characters which are of real systematic importance in a way that each larval stage can be distinguished by a combination of characters shared by most instars (dos Santos \& González-Gordillo, 2004). Furthermore, it has been observed how Processa species do not necessarily pass through every development stage. Some may be omitted while other stages are represented by more than one moult (Gurney, 1923). In this way, it has been found to be appropriate to give a table (Table 2) more than an identification key as it includes morphological characters that otherwise can be ignored while being useful for the correct identification of Processa larvae. When P. macrodactyla is being identified, the characters shown in Table 1 can be useful to determine its zoeal stage.

We wish to thank Dr Antonina dos Santos for constructive comments that improved the initial manuscript. This research was partially supported by post-doctoral fellowships (SFRH/ BPD/1596/2000) from the Ministério da Ciência e da Tecnologia (FCT), Portugal, and by Research Program 'Ramón y Cajal' from the Ministerio de Educación y Ciencia, Spain.

\section{REFERENGES}

Al-Adhub, A.H. \& Williamson, D.I., 1975. Some European Processidae (Crustacea, Decapoda, Caridea). Fournal of Natural History, 9, 693-703.

Anger, K., 2001. The biology of decapod crustacean larvae. The Netherlands: A.A. Balkema Publishers. [Crustacean Issues, no. 14.]

Barnich, R., 1996. The larvae of the Crustacea Decapoda (Excl. Brachyura) in the plankton of the French Mediterranean coast. Göttingen: Cuvillier Verlag.

Bourdillon-Casanova, L., 1960. Le meroplancton du Golfe de Marseille: les larves de Crustacés Décapodes. Recuéil des Travaux de la Station Marine d'Endoume, 30, 1-286.

Clark, P.F., Calazans, D.K. \& Pohle, G.W., 1998. Accuracy and standardization of brachyuran larval descriptions. Invertebrate Reproduction and Development, 33, 127-144.

Fincham, A.A. \& Williamson, D.I., 1978. Crustacea Decapoda: Larvae. VI. Caridea. Fiches d'Identification du Zooplancton, 159/ 160, 1-8.

García Raso, J.E. \& Casanova, C.S., 1985. New record of the species Processa macrodactyla Holthuis, 1952 (Decapoda, Caridea) from Spanish waters. Crustaceana, 49, 88-91.

González-Gordillo, J.I. \& Rodríguez, A., 2000. First larval stage of Scyllarus posteli Forest, 1963 and Processa macrodactyla Holthuis, 1952 hatched in the laboratory. Ophelia, 53, 91-99.

Gurney, R., 1923. The larval stages of Processa canaliculata Leach. Journal of the Marine Biological Association of the United Kingdom, 13, 245-265.

Gurney, R., 1942. Larvae of decapod Crustacea. London: The Ray Society. 
1460 A. Ortega et al. Planktonic stages of Processa macrodactyla

Kurian, G.V., 1956. Larvae of decapod crustacea from the Adriatic Sea. Acta Adriatica, 6, 1-108.

Lebour, M.V., 1936. Notes on the Plymouth Processa (Crustacea). Proceedings of the Zoological Society of London, 1936, 609-617.

Neves, A.M., 1973. Crustáceos Decápodes marinhos de Portugal Continental existentes no Museu Bocage. I. Natantia. Arquivos do Museu Bocage, IV, 71-112.

Nouvel, H. \& Holthuis, L.B., 1957. Les Procesidae (Crustacea, Decapoda, Natantia) des eaux européennes. Zoologische Verhandelingen, 32, 1-53.

Santos, A. dos, 1999. Larvas de crustáceos decápodes ao largo da costa portuguesa. PhD thesis, University of Lisbon, Portugal.

Santos, A. dos \& González-Gordillo, J.I., 2004. Illustrated key for the identification of the Pleocyemata (Crustacea: Decapoda) zoeal stages, from the coastal region of southwestern Europe. Fournal of the Marine Biological Association of the United Kingdom, 84, 205-227.

Smaldon, G., 1993. Coastal shrimps and prawns, 2nd edn. Shrewsbury, UK: Field Studies Council. [Synopses of the British Fauna (New Series), no. 15.]
d'Udekem d'Acoz, C., 1999. Inventaire et distribution des crustacés décapodes de l'Atlantique nord-oriental, de la Méditerranée et des eaux continentales adjacentes au nord de $25^{\circ} \mathcal{N}$. Paris, France: Muséum Nationale d'Historie Naturelle. [Patrimoines Naturels (M.N.H.N./S.P.N.), no. 40.]

Williamson, D.I., 1967. On a collection of planktonic Decapoda and Stomatopoda (Crustacea) from the mediterranean coast of Israel. Bulletin of Sea Fisheries Research Station of Haifa, 45, $32-64$.

Williamson, D.I. \& Rochanaburanon, T., 1979. A new species of Processidae (Crustacea, Decapoda, Caridea) and the larvae of the north European species. Fournal of Natural History, 13, $11-33$.

Submitted 19 May 2005. Accepted 28 September 2005. 